\title{
Measuring microRNA reporter activity in skeletal muscle using hydrodynamic limb vein injection of plasmid DNA combined with in vivo imaging
}

Martin G Guess, Kristen KB Barthel, Emily K Pugach and Leslie A Leinwand ${ }^{*}$

\begin{abstract}
Background: microRNA regulation plays an important role in the remodeling that occurs in response to pathologic and physiologic stimuli in skeletal muscle. In response to stress, microRNAs are dynamically regulated, resulting in a widespread "fine-tuning" of gene expression. An understanding of this dynamic regulation is critical to targeting future therapeutic strategies. Experiments elucidating this dynamic regulation have typically relied on in vitro reporter assays, ex vivo sample analysis, and transgenic mouse studies. Surprisingly, no experimental method to date allows rapid in vivo analysis of microRNA activity in mammals.
\end{abstract}

Methods: To improve microRNA studies we have developed a novel reporter assay for the measurement of skeletal muscle microRNA activity in vivo. To minimize muscle damage, hydrodynamic limb vein injection was used for the introduction of plasmid DNA encoding bioluminescent and fluorescent reporters, including click-beetle luciferase and the far-red fluorescent protein mKATE. We then applied this technique to the measurement of miR-206 activity in dystrophic $m d x 4 C v$ animals.

Results: We found that hydrodynamic limb vein injection is minimally damaging to myofibers, and as a result no induction of muscle-specific miR-206 (indicative of an injury response) was detected. Unlike intramuscular injection or electroporation, we found that hydrodynamic limb vein injection results in dispersed reporter expression across multiple hindlimb muscle groups. Additionally, by utilizing click-beetle luciferase from Pyrophorus plagiophthalamus as a reporter and the far-red fluorescent protein mKATE for normalization, we show as a proof of principle that we can detect elevated miR-206 activity in mdx4CV animals when compared to C57BI/6 controls.

Conclusion: Hydrodynamic limb vein injection of plasmid DNA followed by in vivo bioluminescent imaging is a novel assay for the detection of reporter activity in skeletal muscle in vivo. We believe that this method will allow for the rapid and precise detection of both transcriptional and post-transcriptional regulation of gene expression in response to skeletal muscle stress. Additionally, given the post-mitotic status of myofibers and stable expression of plasmid DNA, we believe this method will reduce biological variability in animal studies by allowing longitudinal studies of the same animal cohort.

Keywords: MicroRNA, In vivo imaging, Hydrodynamic limb vein injection, Plasmid DNA, Reporter assay, Gene therapy, Muscular dystrophy, Luciferase, Bioluminescence

\footnotetext{
* Correspondence: leslie.leinwand@colorado.edu

Department of Molecular, Cellular and Developmental Biology and

BioFrontiers Institute, University of Colorado, Boulder, CO, USA
} 


\section{Background}

Skeletal muscle displays a remarkable ability to remodel in response to pathologic and physiologic stimuli ([1-3] and reviewed in [4]). How changes in gene expression mediate these processes by post-transcriptional regulation has been heavily studied in the context of diseased, hypertrophic, and regenerating skeletal muscle [5-7]. While recent studies have greatly enhanced our understanding of these dynamic processes, measuring posttranscriptional changes that occur in vivo is challenging and this has limited the experimental avenues available. Additionally, the discovery of post-transcriptional regulation by microRNAs (miRNAs) in skeletal muscle has increased the complexity of these processes.

miRNAs are a type of short, non-coding RNA that post-transcriptionally regulate gene expression. miRNAs function by guiding the RNA-induced silencing complex (RISC) to target mRNAs, dictated by base pairing to target sites primarily in their $3^{\prime}$-untranslated regions (UTRs). miRNAs can downregulate the expression of several to hundreds of target genes by repressing translation and/or destabilizing target mRNAs $[8,9]$. miRNAs are required for normal skeletal muscle development in mice [10], and several miRNAs have been shown to be dynamically regulated during hypertrophy [11], acute exercise [12], regeneration after injury [13], and in the remodeling that occurs in response to genetic muscle disease [14-17]. In particular, miR-206 is expressed specifically in skeletal muscle, and is highly expressed in regenerating fibers [13]. miR-206 has been shown to promote terminal differentiation of myoblasts by regulating the expression of genes including connexin43 [18], utrophin [19], pax3 [20], pax7 [21] and DNA polymerase $\alpha$ [22]. Many of these studies rely on data obtained from transgenic mouse studies, and ex vivo sample analysis both currently invaluable to the study of miRNAs. The study of miRNA regulation in skeletal muscle, however, would benefit from a system that enabled rapid, reproducible, longitudinal in vivo reporter assays, at a fraction of the cost of transgenic mouse production and analysis, and with fewer animals needed than for ex vivo studies.

Several studies have shown that stable gene expression can be achieved in post-mitotic myofibers in vivo by the introduction of plasmid DNA (pDNA) [23-26]. Unlike viral DNA vectors, pDNA is non-immunogenic [23], easily manipulated, and relatively inexpensive to produce. Methods for introducing naked pDNA into myofibers include intramuscular injection [24], electroporation $[26,27]$, and more recently, hydrodynamic limb vein (HLV) injection [28,29]. While these methods all result in efficient myofiber transduction, only HLV injection is minimally damaging to the muscle [30], enabling studies of muscle remodeling to take place in the absence of widespread myofiber regeneration. Moreover, HLV injection results in more widespread pDNA distribution than intramuscular injection or electroporation, which are limited to the site of injection, or single muscle groups, respectively $[24,26]$.

In vivo bioluminescent and fluorescent imaging (BLI) of genetically encoded reporters has been a useful tool in murine xenograft studies including cancer [31], chondrogenic differentiation [32], viral infection [33], and even in studies of miRNA biogenesis and posttranscriptional regulation $[34,35]$. All of these studies, however, have relied on the expression of reporter genes in cells cultured in vitro, and none have demonstrated the ability to quantify skeletal muscle reporter gene expression in situ. We therefore developed a novel system using HLV injection of reporter pDNA into skeletal muscle in combination with in vivo BLI in order to quantify miRNA activity. Before testing, we anticipated that animal-to-animal variation in injection efficiency would confound true changes in reporter expression. While several groups have reported the utility of Renilla luciferases as a normalizer in in vivo reporter studies $[32,36]$, we found that the fast kinetics and requirement for intravenous substrate delivery made this approach technically challenging. To solve this problem, we employed a dual-reporter approach consisting of a high-efficiency click-beetle luciferase from Pyrophorus plagiophthalamus (CBG99) [37], and the far-red fluorescent protein mKATE [38] for signal normalization. mKATE has been shown to have a maximum emission wavelength $(635 \mathrm{~nm})$ that is optimal for tissue penetration, and to have high $\mathrm{pH}$ and photostability, making this an ideal protein for in vivo BLI.

As a proof of principle, we measured miR-206 activity and show that the activity measured using this technique reproduces the levels obtained from quantitative reverse transcription PCR (qRT-PCR) measurements. We believe this technique will prove useful not only in the quantification of real-time miRNA activity, but also in studies of transcriptional and post-transcriptional regulation.

\section{Methods}

\section{Plasmid construction}

Two perfectly complementary miR-206 [NCBI:NR_029593] binding sites were inserted between the Xba1 and Fse1 restriction sites downstream of the CBG99 stop codon in pCBG99-Control (Promega, Madison, WI, USA) using the following oligonucleotide sequences: $5^{\prime}$-CTAGACC ACACACTTCCTTACATTCCAAAACCACACACTTCC TTACATTCCAGGCCGG, and 5'-CCTGGAATGTAAG GAAGTGTGTGGTTTTGGAATGTAAGGAAGT GTG TGGT. pcDNA-mKATE was a kind gift from Amy Palmer (University of Colorado at Boulder, Boulder, CO, 
USA), and pCMV-eGFP from Stephen Langer (University of Colorado at Boulder, Boulder, CO, USA).

\section{Hydrodynamic limb vein injection}

All animal experiments were performed using protocols approved by University of Colorado and Colorado State University Institutional Animal Care and Use Committees (IACUC). Mice were injected according to a protocol modified from Hagstrom, et al. [29]. Briefly, after sedation with 1 to $4 \%$ inhaled isoflurane, a tourniquet was secured around the upper hindlimb to restrict blood flow for 1 to 2 minutes prior to injection, and 2 minutes after injection. The hindlimb was first cleaned with $70 \%$ ethanol, and an incision was made with surgical scissors on the medial surface of the leg to expose the great saphenous vein. A $1 / 2$ inch 30 -gauge needle connected by catheter to a syringe was then inserted in an anterograde direction into the vein. Endotoxin-free pDNA isolated using Endofree ${ }^{\bullet}$ Plasmid Maxi Kit (Qiagen, Hilden, Germany) and diluted in sterile saline solution (volume determined according to the formula: $1+(($ body weight in grams -25$) / 25) \times 1 / 2) \mathrm{mL}$ ), was delivered at a rate of $7 \mathrm{~mL} /$ minute by a programmable syringe pump (KD Scientific, Holliston, MA, USA). Two minutes after the injection was completed, the tourniquet was released and the incision was closed with nonabsorbable 6-0 silk suture (Davis-Geck, Brooklyn, NY, USA). Mice recovered on a $37^{\circ} \mathrm{C}$ heat block and were monitored for adverse effects.

\section{Barium chloride injury}

To induce muscle degeneration, mice were first anesthetized with 1 to $4 \%$ inhaled isoflurane, and the right hindlimb was shaved. After cleaning the area with $70 \%$ ethanol, the right gastrocnemius was injected with $50 \mu \mathrm{L}$ of a $1.2 \%$ barium chloride solution in normal saline using a 27-gauge insulin syringe. Mice recovered on a $37^{\circ} \mathrm{C}$ heat block and were monitored for adverse effects.

\section{In vivo bioluminescent and fluorescent imaging}

Animals were anesthetized using 1 to $4 \%$ isoflurane prior to imaging and placed in the chamber of an IVIS 100 in vivo imaging system (Caliper Biosciences, Hopkinton, MA, USA) housed at Colorado State University's Animal Cancer Center (Fort Collins, CO, USA). The mKATE fluorescent signal was then collected using sequential mode with a 1-s exposure time and the Cy5.5 excitation/ emission filter set. Animals were then removed from the imaging chamber and injected intraperitoneally with 200 $\mu \mathrm{L}$ of $30 \mathrm{mg} / \mathrm{mL} \mathrm{D}$-luciferin. Ten minutes after substrate injection, bioluminescent signal was collected for $1 \mathrm{mi}-$ nute using the open filter mode. Images were analyzed using LivingImage software (Caliper Biosciences) and photons $/ \mathrm{sec} / \mathrm{cm}^{2} /$ steradian were quantified using region- of-interest (ROI) analysis. Mice recovered from anesthesia on a $37^{\circ} \mathrm{C}$ heat block.

\section{Immunofluorescence}

Muscles for immunofluorescence were frozen in liquid nitrogen-cooled isopentane and mounted in optimal cutting temperature (OCT) medium (Sakura Finetek, Torrance, CA, USA). Cryosections (12 $\mu \mathrm{m}$ thick) were fixed in $4 \%$ paraformaldehyde for 10 minutes at room temperature, then blocked for 1 hour with $5 \%$ goat serum in PBS and $0.1 \%$ Triton X-100. Following blocking, sections were stained with anti-laminin at 1:500 (L-9393, Sigma, St. Louis, MO, USA) or anti-desmin at 1:20 (D-8281, Sigma) overnight at $4^{\circ} \mathrm{C}$. After washing several times in PBS with $0.1 \%$ Triton X-100, anti-rabbit Texas red secondary antibody was applied at 1:100 dilution for 1 hour at $37^{\circ} \mathrm{C}$ (711-075-152, Jackson, West Grove, PA, USA). After washing again in PBS with $0.1 \%$ Triton X-100, sections were counterstained with $300 \mathrm{nM}$ 4',6-diamidino-2-phenylindole (DAPI) for 5 minutes at room temperature (Sigma D-9542), and mounted using Fluoromount G (Southern Biotech, Birmingham, AL, USA).

\section{Quantification of injection efficiency}

To determine HLV injection efficiency, percentages of GFP-positive fibers were quantified using immunofluorescence on lower hindlimb sections from pCMV-eGFPinjected animals. Following imaging on an inverted epifluorescent microscope (Eclipse TE2000, Nikon, Melville, NY, USA), GFP, laminin, and DAPI images were merged to produce a composite (ImageJ software, National Institutes of Health, Bethesda, MD, USA). GFP-positive fibers were counted when GFP signal across a myofiber was greater than background levels. Total fiber number was counted using laminin staining to demarcate fiber boundaries. Percentages are reported as an average of GFP-positive fibers for ten fields of view for the gastrocnemius, and five fields each for the soleus and tibialis anterior (TA).

\section{qRT-PCR}

To measure miRNA expression, total RNA was first isolated from snap-frozen skeletal muscles using TRI reagent (Molecular Research Center, Cincinnati, OH, USA). Then, 7 ng of total RNA per reaction was reversetranscribed and PCR-amplified on a CFX96 thermocycler (Bio-Rad, Hercules, CA, USA) using Taqman miRNA assays (Invitrogen, Grand Island, NY, USA). Relative miRNA expression was determined using the $2^{-\Delta \Delta \mathrm{Ct}}$ method [39], using sno202 as a reference gene. 


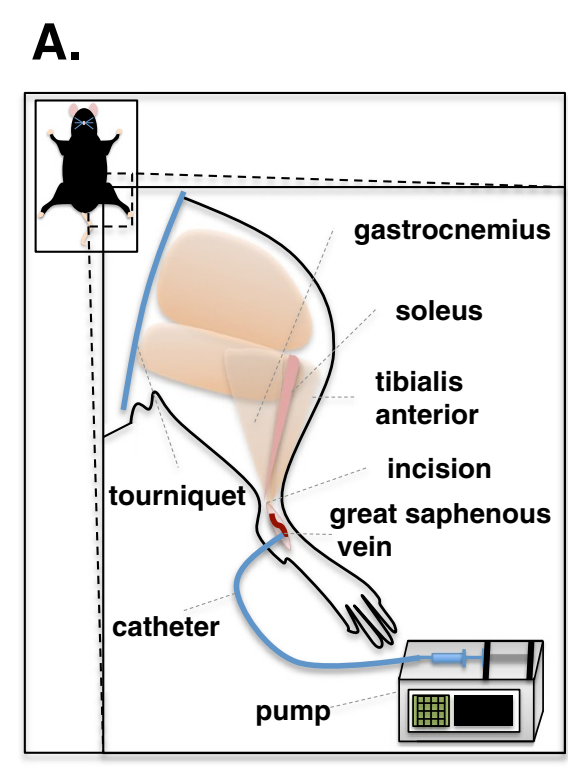

B.

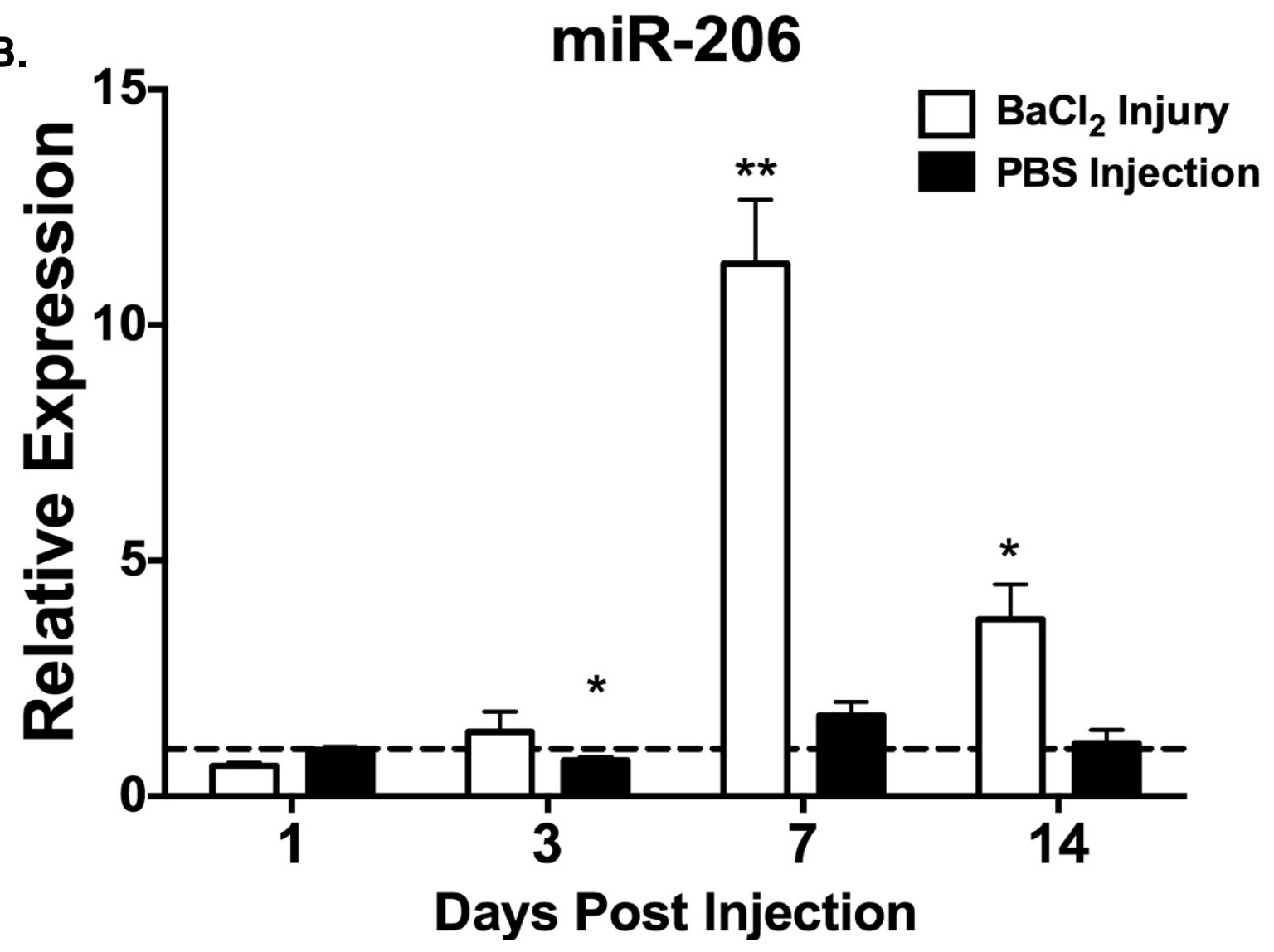

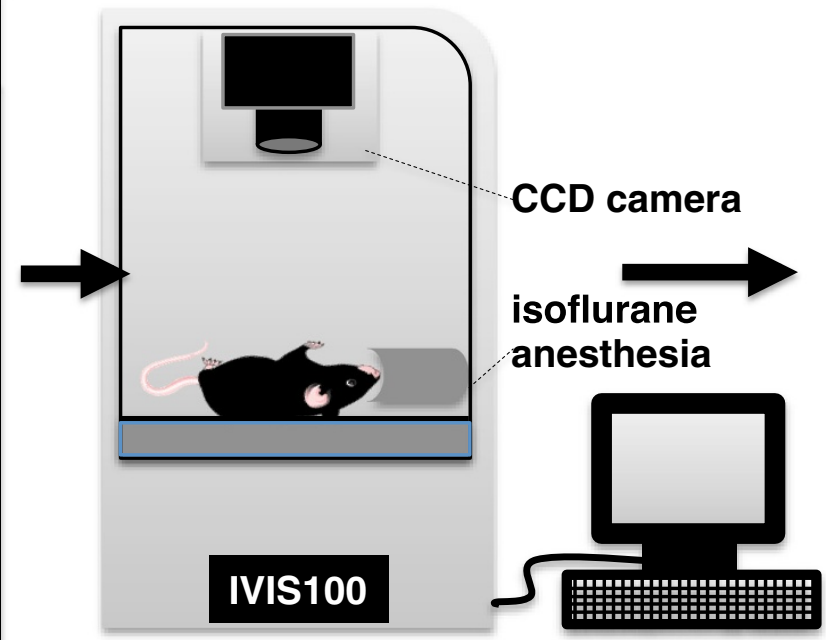

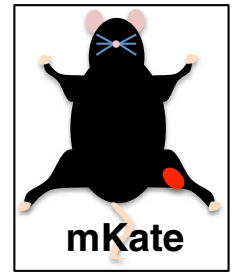

inject luciferin

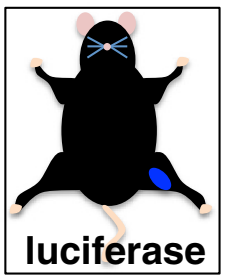

Figure 1 miR-206 levels after hydrodynamic limb vein injection. (A) Schematic depiction of hydrodynamic limb vein injection (HLV) of plasmid DNA followed by in vivo bioluminescent and fluorescent imaging (BLI). (B) miR-206 expression in 3 to 4 month-old C57Bl/6 mice receiving either $\mathrm{HLV}$ injection of saline solution or $\mathrm{BaCl}_{2}$ injury, sacrificed at the indicated time points, normalized to sno202. Relative levels measured in the right (treated) gastrocnemius are displayed as mean values normalized to contralateral controls; $n=3$ or 4 animals/group, Error bars $=$ standard error of the mean. ${ }^{*} P \leq 0.05,{ }^{* *} P \leq 0.001$. 


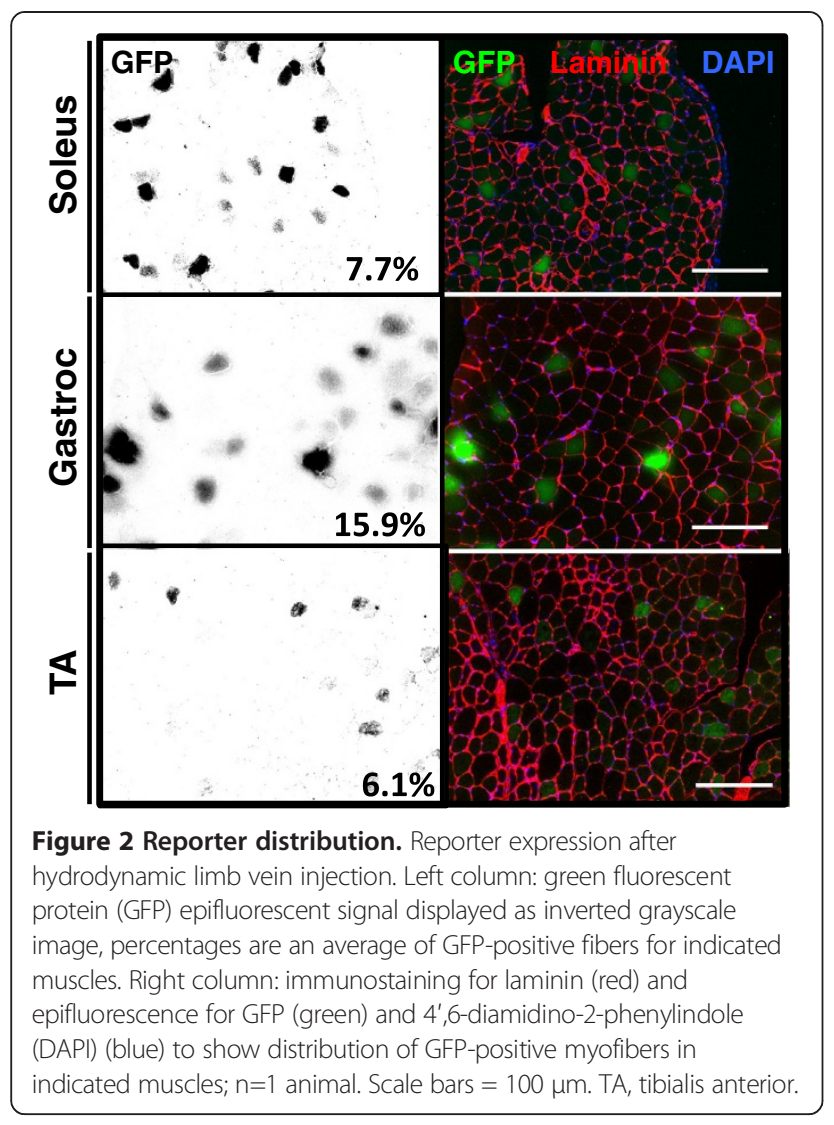

\section{Statistical analysis}

For qRT-PCR experiments, relative miR-206 expression values were compared to controls (for assessment of muscle damage, controls were uninjured/uninjected contralateral limbs for each time point, and for miR-206 reporter measurements, controls were $\mathrm{C} 57 \mathrm{Bl} / 6$ animals) using the unpaired Student's $t$-test. For BLI measurements, statistics were performed by comparing mean normalized photons $/ \mathrm{s} / \mathrm{cm}^{2} /$ steradian (CBG99/mKATE) of pCBG99-2x-miR-206-injected hindlimbs, to mean normalized photons $/ \mathrm{s} / \mathrm{cm}^{2} /$ steradian of pCBG99-Controlinjected hindlimbs for $m d x 4 c v$ and $\mathrm{C} 57 \mathrm{Bl} / 6$ animals using the unpaired Student's $t$-test.

\section{Results}

HLV injection does not induce myofiber regeneration

Prior to employing HLV to deliver miRNA reporters for miR-206 activity, it was important to determine whether the injection technique itself would induce muscle regeneration and consequently increase the levels of miR-206. To test this, we performed HLV injection (Figure 1A) on wild-type $\mathrm{C} 57 \mathrm{Bl} / 6$ mice using saline only and compared miR-206 expression to that in animals injured with $\mathrm{BaCl}_{2}$. miR-206 levels decreased slightly $(P=0.02)$ on the third day after saline injection, likely due to residual edema from the high-volume injection, but remained unchanged (day 7 animals trended towards a 1.7-fold

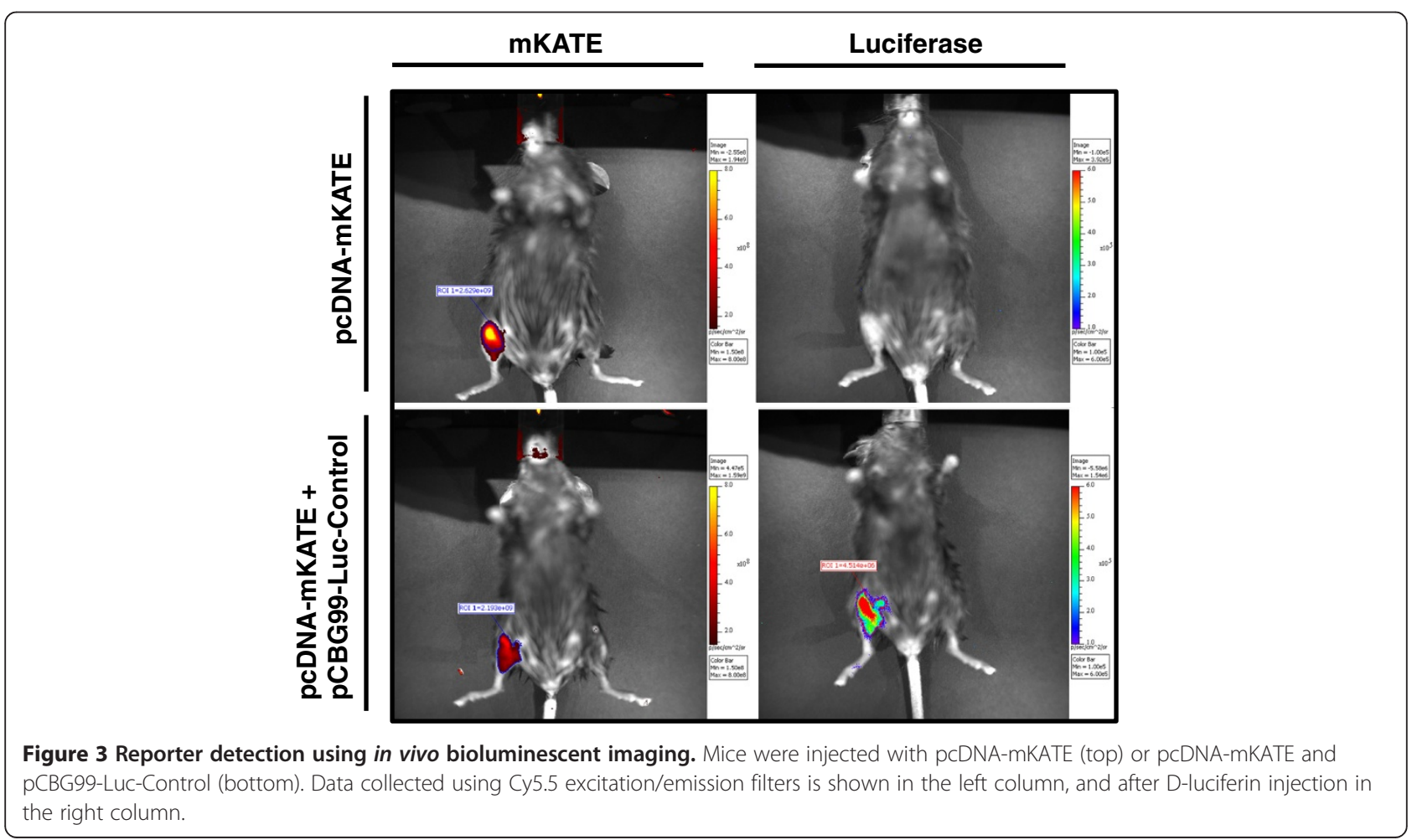




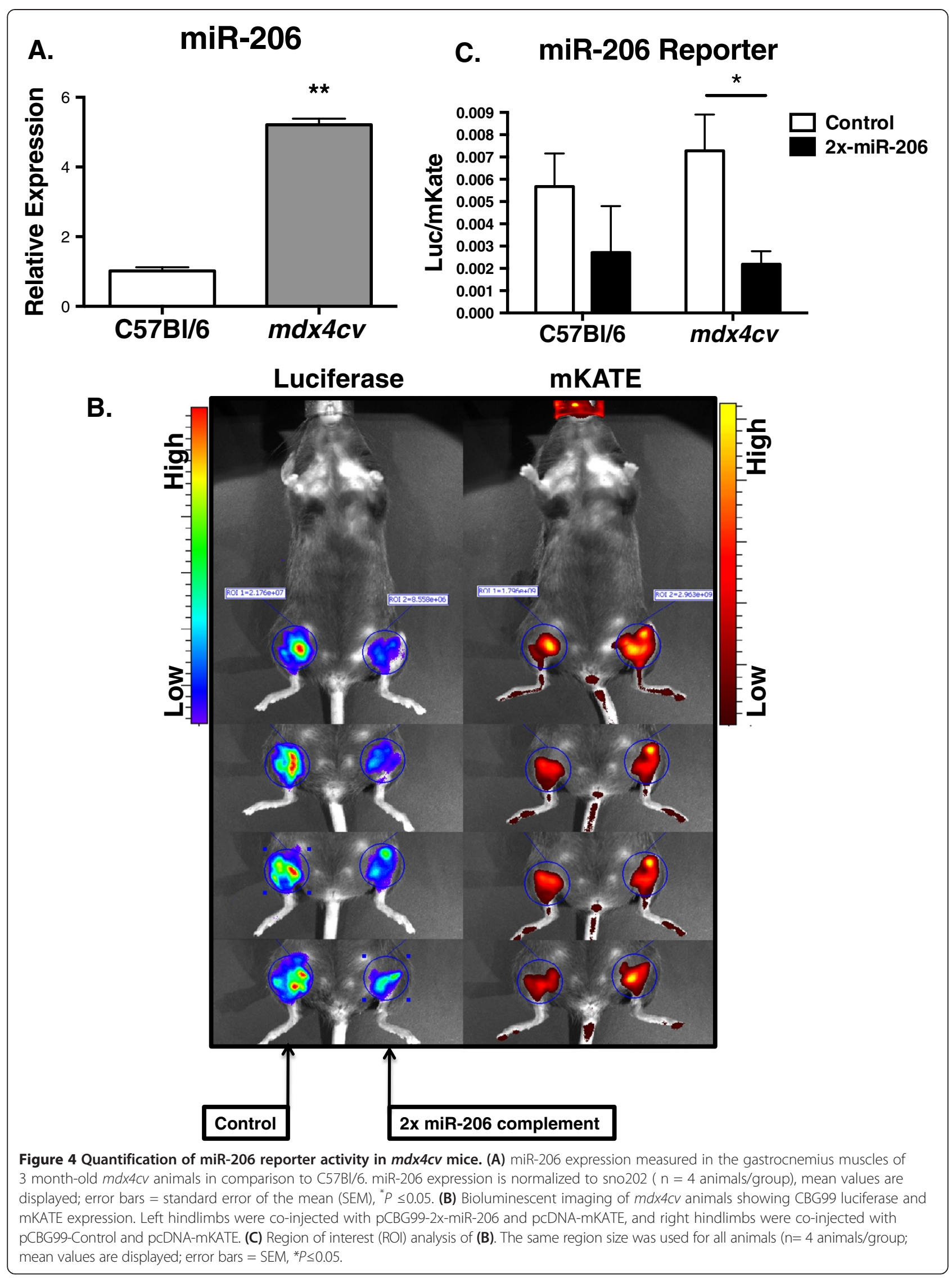


increase, $P=0.051$ ) on subsequent days (Figure 1B). Conversely, barium chloride injury strongly induced miR-206 approximately 11 -fold $(P=0.0003)$ as previously reported [13], suggesting that HLV injection does not activate a widespread program of muscle regeneration.

Reporter expression is well distributed and is quantifiable using in vivo imaging

To test the muscle distribution of reporter pDNA, we next injected C57Bl/6 mice with $100 \mu \mathrm{g} / \mathrm{animal}$ of pCMV-eGFP. After 7 days, we performed immunofluorescence on fixed cryosections and found that GFP expression was visible in the sarcoplasm of myofibers in the gastrocnemius, soleus, and TA muscles (Figure 2). To determine injection efficiency, we quantified GFPpositive myofibers and found that $7.7 \%, 15.9 \%$ and $6.1 \%$ of fibers in the soleus, gastrocnemius, and TA, respectively, expressed the reporter. We also observed an absence of centrally located nuclei in GFP-positive myofibers, further supporting the finding that HLV injection itself causes minimal muscle injury. Next, we tested whether reporter gene expression can be quantified from both bioluminescent and fluorescent reporters using in vivo imaging. To this end, we injected either $100 \mu \mathrm{g}$ each of pCBG99-Luc-Control and pcDNAmKATE, or pcDNA-mKATE alone into the right hindlimbs of $\mathrm{C} 57 \mathrm{Bl} / 6$ mice and collected images 7 days later using BLI. The signal was easily visible and localized to the hindlimb skeletal muscle for both reporters, although mKATE background signal was frequently observed from the ventilation nosepiece and/or the distal parts of the hindlimb (Figure 3). We also found that mKATE fluorescence did not bleed into the luciferase channel, making it ideal for in vivo use in combination with CBG99-luciferase.

\section{miRNA activity measurements are consistent with miRNA} qRT-PCR measurements

In order to measure miRNA activity in vivo, we inserted two perfectly complementary miRNA binding sites downstream of the CBG99 luciferase coding sequence, creating pCBG99-2x-miR-206. We next co-injected $100 \mu \mathrm{g}$ of pcDNA-mKATE along with either pCBG99-2x-miR-206 (left hindlimbs) or pCBG99-Luc-Control (right hindlimbs) into $\mathrm{C} 57 \mathrm{Bl} / 6$ or dystrophic $m d x 4 c v$ mice. Seven days later, we measured the signal using in vivo BLI followed by ROI analysis (Figure 4B). To ensure that we minimized the effect of differential plasmid distribution and injection efficiency, the same region size was used for quantification in all animals, and the luciferase signal was normalized to mKATE. After normalizing, the CBG99:mKATE ratio from left hindlimbs was compared to control right hindlimbs. In agreement with a 5-fold increase in miR-206 expression in $m d x 4 c v$ animals by qRT-PCR $(P=0.0001)$

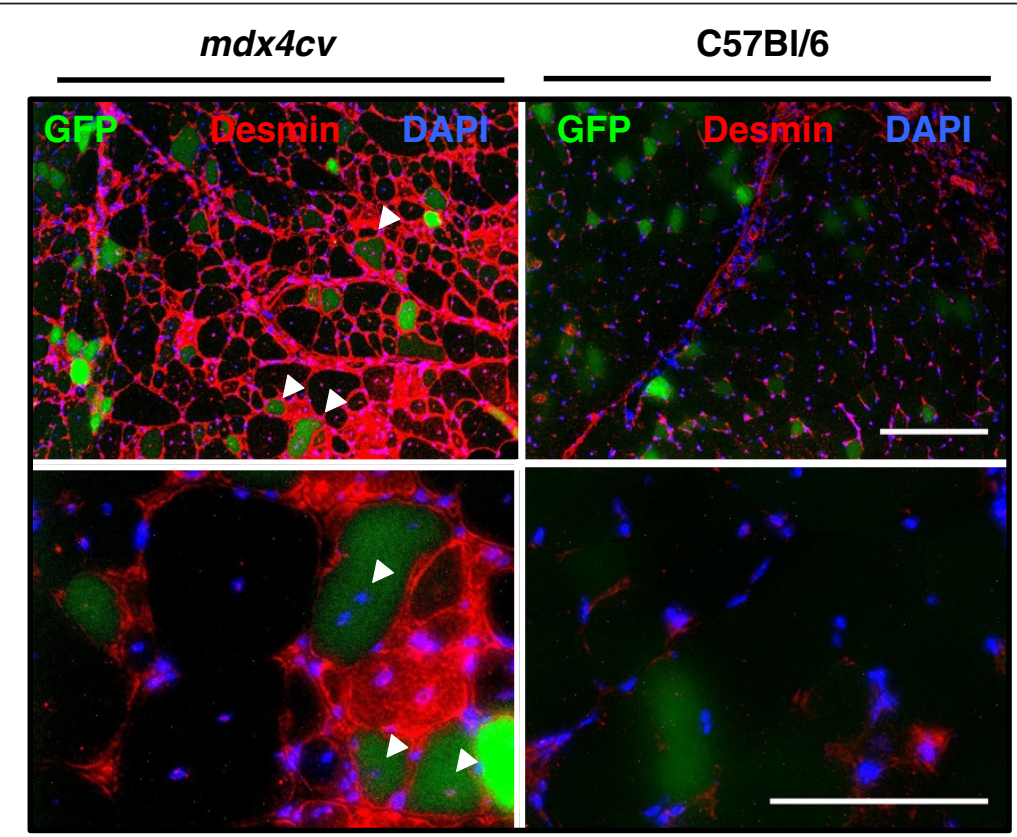

Figure 5 Reporter expression in regenerating $\mathbf{m d x} \mathbf{4} \boldsymbol{c v}$ fibers. pCMV-eGFP-injected $m d x 4 c v$ (left column) and C57BI/6 (right column) gastrocnemius sections showing desmin immunostaining (red), green fluorescent protein (GFP) epifluorescence (green), and 4',6-diamidino-2phenylindole (DAPI) (blue) demonstrate GFP expression in regenerating fibers. Upper row images were taken using 10x objective lens, lower row with $20 \times$ objective. Scale bars $=100 \mu \mathrm{m}$. 
(Figure 4A), the normalized bioluminescent signal in the left hindlimbs of $m d x 4 c v$ mice was reduced 3.3-fold $(P=0.02)$ (Figure $2 C)$. While the decreased average signal in $\mathrm{C} 57 \mathrm{Bl} / 6$ did not reach significance $(P=0.15)$, the downward trend is likely due to the high abundance of miR-206 in skeletal muscle, and the decreased miR-206 reporter signal in $m d x 4 c v$ animals is due to increased miR-206 expression. Given that miR-206 is highly expressed in the regenerating fibers of dystrophic mice [13], this result suggests that pDNA injected using HLV is also expressed in these fibers. To test this, we injected $m d x 4 c v$ animals with $100 \mu \mathrm{g}$ of pCMV-eGFP and collected tissues for immunofluorescence 7 days later. As expected, we found GFP expression in small, desmin-positive, regenerating myofibers with centrally located nuclei (Figure 5, arrowheads), indicating that the decrease in luciferase signal measured using BLI likely corresponds to a loss of luciferase activity in these fibers.

\section{Discussion}

Several groups have reported the safety and efficacy of pDNA injections using HLV for gene therapy approaches $[23,28,29]$. We found this technique also to be useful in studies requiring minimally damaging introduction of genetically encoded reporters into skeletal muscle and have used it in combination with BLI and ROI analysis to analyze $M y h 7 b$ promoter activity in vivo [40]. We also found that this technique induces minimal muscle regeneration as assessed by miR-206 induction, and to our knowledge this is the first report of using an in vivo transfection method that does not induce myofiber regeneration for luciferase reporter studies.

The distribution of GFP-positive myofibers after HLV injection that we have seen is similar to that reported by Wooddell, et al. [41] although the percentage of transfected fibers we report is lower. This may be due to decreased sensitivity of detecting a fluorescent reporter (GFP) versus a colorimetric stain ( $\beta$-galactosidase). Similar to this report, we find the highest percentage (15.9\%) of transfected myofibers in the posterior lower leg (gastrocnemius) muscles and the lowest percentage (6.1\%) in the anterior lower leg (TA) muscles (Figure 3). Additionally, others have observed reporter gene expression up to 49 weeks after HLV injection [41], suggesting that this method could be used for longer-term regulatory studies, provided that the luciferase and mKATE signals follow similar expression profiles.

\section{Conclusion}

In summary, HLV injection of pDNA reporters into skeletal muscle followed by BLI is a useful technique for studies of gene regulation. Here, we show that it is possible to measure miRNA activity in myofibers in situ, without activation of a regeneration response. This technique has the added benefit of reducing the cost associated with producing transgenic animals for skeletal muscle studies, and will likely allow reduced animal numbers and decreased variability in future experiments by enabling longitudinal studies of the same animal cohort.

\section{Abbreviations}

BLI: Bioluminescent and fluorescent imaging; DAPI: 4',6-diamidino-2phenylindole; GFP: Green fluorescent protein HLV: hydrodynamic limb vein; miRNA: microRNA; OCT: Optimum cutting temperature; PBS: Phosphatebuffered saline; pDNA: Plasmid DNA; qRT-PCR: Quantitative reverse transcription polymerase chain reaction; RISC: RNA-induced silencing complex; ROI: Region of interest; UTR: Untranslated region; TA: Tibialis anterior.

\section{Competing interests}

The authors have no competing interests to declare.

\section{Authors' contributions}

MG designed the experiments, wrote the paper, and carried out the qRT-PCR, injections, imaging, and molecular cloning. KB played a key role in the project's conception and provided useful discussion and critical reading of the manuscript. EP provided assistance with in vivo BLI and assembling figures. LL provided mentoring and discussion about all experiments and data. All authors have read and approved the manuscript.

\section{Acknowledgements}

We would like to thank Mark Noble for assistance with HLV injection, Adam Chicco for assistance with animal protocols, and Margaret Isenhart for animal care. This work was funded by NIH R01GM029090 to LL and NIH 5T32GM007135-36 to MG.

Received: 13 February 2013 Accepted: 28 May 2013

Published: 1 August 2013

\section{References}

1. Porter JD, Khanna S, Kaminski HJ, Rao JS, Merriam AP, Richmonds CR, Leahy $P, L i ~ J, G u o$ W, Andrade FH: A chronic inflammatory response dominates the skeletal muscle molecular signature in dystrophin-deficient $\mathrm{mdx}$ mice. Hum Mol Genet 2002, 11:263-272.

2. Haddad F, Herrick RE, Adams GR, Baldwin KM: Myosin heavy chain expression in rodent skeletal muscle: effects of exposure to zero gravity. J Appl Physiol 1993, 75:2471-2477.

3. Daugaard JR, Nielsen JN, Kristiansen S, Andersen JL, Hargreaves M, Richter EA: Fiber type-specific expression of GLUT4 in human skeletal muscle: influence of exercise training. Diabetes 2000, 49:1092-1095.

4. Bassel-Duby R, Olson EN: Signaling pathways in skeletal muscle remodeling. Annu Rev Biochem 2006, 75:19-37.

5. Farina NH, Hausburg M, Dalla Betta N, Pulliam C, Srivastava D, Cornelison DD, Olwin BB: A role for RNA post-transcriptional regulation in satellite cell activation. Skeletal Muscle 2012, 2:1

6. Mahadevan M, Tsilfidis C, Sabourin L, Shutler G, Amemiya C, Jansen G, Neville C, Narang M, Barceló J, O'Hoy K: Myotonic dystrophy mutation: an unstable CTG repeat in the $3^{\prime}$ untranslated region of the gene. Science 1992, 255:1253-1255.

7. Cesana M, Cacchiarelli D, Legnini I, Santini T, Sthandier O, Chinappi M, Tramontano A, Bozzoni I: A long noncoding RNA controls muscle differentiation by functioning as a competing endogenous RNA. Cell 2011, 147:358-369.

8. Djuranovic S, Nahvi A, Green R: MiRNA-mediated gene silencing by translational repression followed by mRNA deadenylation and decay. Science 2012, 336:237-240.

9. Bazzini AA, Lee MT, Giraldez AJ: Ribosome profiling shows that miR-430 reduces translation before causing mRNA decay in zebrafish. Science 2012, 336:233-237.

10. O'Rourke JR, Georges SA, Seay HR, Tapscott SJ, McManus MT, Goldhamer DJ, Swanson MS, Harfe BD: Essential role for Dicer during skeletal muscle development. Dev Biol 2007, 311:359-368. 
11. McCarthy JJ, Esser KA: MicroRNA-1 and microRNA-133a expression are decreased during skeletal muscle hypertrophy. J Appl Physiol 2007, 102:306-313.

12. Safdar A, Abadi A, Akhtar M, Hettinga BP, Tarnopolsky MA, Lucia A: miRNA in the regulation of skeletal muscle adaptation to acute endurance exercise in C57BI/6J male mice. PLoS One 2009, 4:e5610-e5610.

13. Yuasa K, Hagiwara Y, Ando M, Nakamura A, Takeda S, Hijikata T: MicroRNA206 is highly expressed in newly formed muscle fibers: implications regarding potential for muscle regeneration and maturation in muscular dystrophy. Cell Struct Funct 2008, 33:163-169.

14. McCarthy JJ, Esser KA, Andrade FH: MicroRNA-206 is overexpressed in the diaphragm but not the hindlimb muscle of $\mathrm{mdx}$ mouse. Am J Physiol Cell Physiol 2007, 293:C451-C457.

15. Liu N, Williams AH, Maxeiner JM, Bezprozvannaya S, Shelton JM, Richardson JA, Bassel-Duby R, Olson EN: microRNA-206 promotes skeletal muscle regeneration and delays progression of Duchenne muscular dystrophy in mice. J Clin Invest 2012, 122:2054-2065.

16. Eisenberg I, Eran A, Nishino I, Moggio M, Lamperti C, Amato AA, Lidov HG, Kang PB, North KN, Mitrani-Rosenbaum S, Flanigan KM, Neely LA, Whitney $D$, Beggs AH, Kohane IS, Kunkel LM: Distinctive patterns of microRNA expression in primary muscular disorders. Proc Natl Acad Sci USA 2007, 104:17016-17021.

17. Gambardella S, Rinaldi F, Lepore SM, Viola A, Loro E, Angelini C, Vergani L, Novelli G, Botta A: Overexpression of microRNA-206 in the skeletal muscle from myotonic dystrophy type 1 patients. J Trans/ Med 2010, 8:48.

18. Anderson $\mathrm{C}$, Catoe $\mathrm{H}$, Werner R: MIR-206 regulates connexin43 expression during skeletal muscle development. Nucleic Acids Res 2006 34:5863-5871.

19. Rosenberg MI, Georges SA, Asawachaicharn A, Analau E, Tapscott SJ: MyoD inhibits Fstl1 and Utrn expression by inducing transcription of miR-206. J Cell Biol 2006, 175:77-85.

20. Boutet SC, Cheung TH, Quach NL, Liu L, Prescott SL, Edalati A, lori K, Rando TA: Alternative polyadenylation mediates microRNA regulation of muscle stem cell function. Cell Stem Cell 2012, 10:327-336.

21. Dey BK, Gagan J, Dutta A: MiR-206 and -486 induce myoblast differentiation by downregulating Pax7. Mol Cell Biol 2010, 31:1-46.

22. Kim HK, Lee YS, Sivaprasad U, Malhotra A, Dutta A: Muscle-specific microRNA miR-206 promotes muscle differentiation. J Cell Biol 2006, 174:677-687.

23. Sebestyen MG, Hegge JO, Noble M, Lewis D, Herweijer H, Wolff JA: Progress toward a non-viral gene therapy protocol for the treatment of anemia. Hum Gene Ther 2007, 18:269-285.

24. Wolff JA, Malone RW, Williams P, Chong W, Acsadi G, Jani A, Felgner PL: Direct gene transfer into mouse muscle in vivo. Science 1990, 247:1465-1468.

25. Fabre EE, Bigey $P$, Orsini C, Scherman D: Comparison of promoter region constructs for in vivo intramuscular expression. J Gene Med 2006, 8:636-645.

26. Aihara H, Miyazaki J-l: Gene transfer into muscle by electroporation in vivo. Nat Biotechnol 1998, 16:867-870.

27. Deleuze $V$, Scherman D, Bureau MF: Interleukin-10 expression after intramuscular DNA electrotransfer: kinetic studies. Biochem Biophys Res Commun 2002, 299:29-34.

28. Suda T, Liu D: Hydrodynamic gene delivery: its principles and applications. Mol Ther 2007, 15:2063-2069.

29. Hagstrom JE, Hegge J, Zhang G, Noble M, Budker V, Lewis DL, Herweijer H, Wolff JA: A facile nonviral method for delivering genes and siRNAs to skeletal muscle of mammalian limbs. Mol Ther 2004, 10:386-398.

30. Toumi H, Hegge J, Subbotin V, Noble M, Herweijer H, Best TM, Hagstrom JE: Rapid intravascular injection into limb skeletal muscle: a damage assessment study. Mol Ther 2006, 13:229-236.

31. Kim HJ, Chung J-K, Hwang DW, Lee DS, Kim S: In vivo imaging of miR-221 biogenesis in papillary thyroid carcinoma. Mol Imaging Biol 2009, 11:71-78.

32. Vilalta M, Jorgensen C, Degano IR, Chernajovsky Y, Gould D, Noel D, Andrades JA, Becerra J, Rubio N, Blanco J: Dual luciferase labelling for noninvasive bioluminescence imaging of mesenchymal stromal cell chondrogenic differentiation in demineralized bone matrix scaffolds. Biomaterials 2009, 30:4986-4995.
33. Luker KEK, Luker GDG: Applications of bioluminescence imaging to antiviral research and therapy: multiple luciferase enzymes and quantitation. Antiviral Res 2008, 78:9-9.

34. Lee JY, Kim S, Hwang DW, Jeong JM, Chung J-K, Lee MC, Lee DS: Development of a dual-luciferase reporter system for in vivo visualization of MicroRNA biogenesis and posttranscriptional regulation. J Nucl Med 2008, 49:285-294.

35. Kim S, Hwang DW, Lee DS: A study of microRNAsin silicoandin vivo: bioimaging of microRNA biogenesis and regulation. FEBS J 2009, 276:2165-2174.

36. Bhaumik S, Gambhir SS: Optical imaging of Renilla luciferase reporter gene expression in living mice. Proc Natl Acad Sci USA 2002, 99:377-382.

37. Miloud T, Henrich C, Hämmerling GJ: Quantitative comparison of click beetle and firefly luciferases for in vivo bioluminescence imaging. J Biomed Opt 2007, 12:054018.

38. Shcherbo D, Merzlyak EM, Chepurnykh TV, Fradkov AF, Ermakova GV, Solovieva EA, Lukyanov KA, Bogdanova EA, Zaraisky AG, Lukyanov S, Chudakov DM: Bright far-red fluorescent protein for whole-body imaging. Nat Methods 2007, 4:741-746.

39. Livak KJ, Schmittgen TD: Analysis of relative gene expression data using real-time quantitative $P C R$ and the 2(-Delta Delta $C(T)$ ) Method. Methods 2001, 25:402-408.

40. Yeung F, Chung E, Guess MG, Bell ML, Leinwand LA: Myh7b/miR-499 gene expression is transcriptionally regulated by MRFs and Eos. Nucleic Acids Res 2012, 40:7303-7318.

41. Wooddell $\mathrm{Cl}$, Hegge JO, Zhang G, Sebestyén MG, Noble M, Griffin JB, Pfannes LV, Herweijer H, Hagstrom JE, Braun S, Huss T, Wolff JA: Dose response in rodents and nonhuman primates after hydrodynamic limb vein delivery of naked plasmid DNA. Hum Gene Ther 2011, 22:889-903.

doi:10.1186/2044-5040-3-19

Cite this article as: Guess et al:: Measuring microRNA reporter activity in skeletal muscle using hydrodynamic limb vein injection of plasmid DNA combined with in vivo imaging. Skeletal Muscle 2013 3:19.

\section{Submit your next manuscript to BioMed Central and take full advantage of:}

- Convenient online submission

- Thorough peer review

- No space constraints or color figure charges

- Immediate publication on acceptance

- Inclusion in PubMed, CAS, Scopus and Google Scholar

- Research which is freely available for redistribution

Submit your manuscript at www.biomedcentral.com/submit
C) Biomed Central 